\title{
Proceeding
}

Supplementary Issue: Spring Conferences of Sports Science. Costa Blanca Sports Science Events, 19-20 June 2020. Alicante, Spain.

\section{Analysis of ball interception velocity in futsal goalkeepers}

\author{
MAURO CALLEJAS-CUERVO ${ }^{1} \triangle$, JAIRO A. PINEDA-ROJAS², WILLIAM A. DAZA-WITTINGHAN ${ }^{3}$ \\ ${ }^{1}$ School of Computer Science, Software Research Group, Pedagogical and Technological University of \\ Colombia, Colombia \\ 2Pan American College of Puerto Boyacá, Secretary of Education of Boyacá, Colombia \\ ${ }^{3}$ School of Physical Education and Recreation and Sports, Pedagogical Trends Group, Pedagogical and \\ Technological University of Colombia, Colombia
}

\begin{abstract}
Technology constitutes a support to sports training which has an influence on the preparation of the player. This article reports on the analysis of the ball interception velocity in futsal goalkeepers. For this, a tool was created based on inertial and magnetic sensors, and a contact platform which permitted the analysis of the interception velocity. Then, a procedure was designed to execute a number of tests and a series of trials were carried out to define the factors to be evaluated. After that, tests were applied to 10 goalkeepers from the Liga Colombiana de Fútbol de Salón (Colombian Futsal League), which would permit the measurement of the study variable. The anthropometric characteristics presented by the goalkeepers selected were similar according to age, weight and body mass index, allowing homogeneity in the samples. The results showed that $50 \%$ of the interceptions to the right of the goalkeeper had a Low velocity (between 30 and $75 \mathrm{~km} / \mathrm{h}$ ); 40\% were Medium (between 75 and $119 \mathrm{Km} / \mathrm{h}$ ) and 10\% were Fast (more than $119 \mathrm{Km} / \mathrm{h}$ ). In addition, with respect to velocity to the left of the goalkeeper, $40 \%$ were in the Low range (between 32 and $75 \mathrm{Km} / \mathrm{h}$ ) and $60 \%$ in the Medium range $(75$ and $119 \mathrm{Km} / \mathrm{h}$ ). These results show that the goalkeepers have a better velocity performance to their right. It can be concluded that the platform is a precise and ergonomic means to measure the ball interception velocity in goalkeepers.

Keywords: Interception speed; Futsal; Inertial and magnetic sensors; Motion capture; Sport performance.

\section{Cite this article as:}

Callejas-Cuervo, M., Pineda-Rojas, J.A., \& Daza-Wittinghan, W.A. (2020). Analysis of ball interception velocity in futsal goalkeepers. Journal of Human Sport and Exercise, 15(3proc), S735-S747. doi:https://doi.org/10.14198/jhse.2020.15.Proc3.24

Corresponding author. School of Computer Science, Software Research Group, Pedagogical and Technological University of Colombia, Avenida Central del Norte 39-115, 150002, Tunja, Boyacá, Colombia. https://orcid.org/0000-0001-9894-8737

E-mail: mauro.callejas@uptc.edu.co

Supplementary Issue: Spring Conferences of Sports Science. Costa Blanca Sports Science Events, 19-20 June 2020. Alicante, Spain.

JOURNAL OF HUMAN SPORT \& EXERCISE ISSN 1988-5202

(c) Faculty of Education. University of Alicante

doi:10.14198/jhse.2020.15.Proc3.24
\end{abstract}




\section{INTRODUCTION}

Futsal is a famous sport in Colombia and around the world. It has awarded Colombia three world championships and, owing to its characteristics, it can be practised in every corner of the country. Consequently, it is one of the disciplines with the greatest presence, where the demand in its training is related to the specificity of each one of the characteristic positions of this discipline. Among the most distinct and special positions is that of the goalkeeper, with a countless number of physical, technical, and psychological characteristics, which include functional aspects in the practice of the game, which are fundamental to the development of skills and their execution. Thus, it is important to increase the number of investigations that inquire directly into the variables which influence the development of the activities of goalkeepers and contribute to the direction, control and special training of this position.

The goalkeeper is particularly required to show improvement through the training of the basic abilities they possess. Among the elements that have to be exercised is interception, which is related with the speed of the execution of the movement that the goalkeeper uses in order to prevent the ball from entering the net (score a goal). Because of this the direction of the training is dominated by this focus, so much so that it is relevant to add tools that consider more factors in the real practice of game situations and to include technology as a determining factor in the functional structure of the futsal training programmes and plans. Among the studies consulted, there are some that investigate the measurement and analysis of velocity using technological tools as support, in order to evaluate this variable. For example, Navia (2013), in his doctoral thesis, explains the use of some elements that relate visual patterns of the futsal goalkeeper with technological instruments, such as a pupilometer. In this study, 12 goalkeepers participated and the focus was on the trajectory of the ball for its interception. As a consequence, it was concluded that the goalkeepers improved their interception at a 10-meter shot distance and that at a 6-meter distance the difference was not significant. In another doctoral thesis (Antúnez, 2003), the interception of the ball in handball was analysed by means of a video recorder. The aim of the study was to measure the effectiveness of the female handball goalkeepers in the interception of the ball coming from any direction. Video cameras and a video recorder were used to analyse the movements and, thus, create a perceptive motion programme in order to enhance the goalkeepers' interception. The conclusion of this study was that the goalkeepers improved their level of efficiency in the interception of the ball once the aforementioned programme was applied.

In addition, the doctoral work by Martinez (2003) used a body reaction time meter, with the SuperLap programme, which measures reaction time. For this, a task was created in which the individual had to respond with the PC keyboard to an image that appeared on the screen. The time reaction test consisted of pressing a button as fast as possible, when a black square appeared on the screen. From this study, it was concluded that the reaction time is not a completely independent quality, but that it is correlated with movement frequency and manual strength. Finally, Suárez (2010) implemented a platform to measure reaction speed in the Bandal-Chagui technique, among young taekwondo athletes, where the reaction time, execution and frequency of impact were registered. Finally, other research works reported the evaluation of different factors, such as the distance covered in a game, individualised thresholds to analyse the demand of acceleration in football players, using a GPS (Nuñez et al., 2019; Estrada Ruiz, 2019), and also, a new tool to control and monitor the training time of football players (Carlos-Vivas et al., 2019; Ferley et al., 2020; McFadden et al., 2020).

Based on the studies described above, which show the few works carried out up to the present which are focused on the analysis of futsal goalkeepers' performances, it was the aim of this project to conduct a study that was directly linked to the goalkeeper, which allowed the analysis of the interception of the ball, with the 
help of a motion capture system (IMOCAP). This would allow the registration of the precise moment in which the ball was kicked up to the moment of its impact against the goalkeeper.

This article contains four sections. Firstly, the research methodology used for the analysis of the ball interception velocity in futsal goalkeepers is explained. Secondly, the main results of the investigation are presented. Thirdly, there is a discussion of the results. And finally, the conclusions are expressed in the fourth section.

\section{METHODOLOGY}

Within this section, there is a description of the study design as well as the population, sampling, variables, materials used, testing scenario, and the respective procedure.

\section{Study design}

This is a descriptive-correlational study as, initially, it is intended to identify the properties, characteristics, and profiles of the goalkeepers from the futsal teams of the professional championship in Colombia. It is correlational because these types of studies have the purpose of measuring the degree of the relation between two or more concepts or variables, which measures each of them and then, that relationship is quantified and analysed.

\section{Research approach}

This research presents a quantitative approach and has its grounds in the hypothetic-deductive method, given that theories and initial research questions are established, from which different hypotheses are derived. These are tested using appropriate investigation designs. The approach measures the variables in a determined context, analyses the measurements, and draws conclusions. If the results corroborate the hypotheses, the theory is reliable; if not, it is refuted and discarded to look for better ones. On the other hand, it is reductionist, as it uses numerical measurement, counting and statistics, surveys, experimentation, patterns, and data collection.

\section{Population}

It was composed of the players (goalkeepers) of the professional futsal championship in Colombia called: Copa Hero Claro de Fútbol de Salón (Claro Futsal Hero Cup). At the moment of the study, a total of 20 teams had enrolled, each one with 2 goalkeepers registered (a starting player and a substitute).

\section{Sample}

A sample of 10 professional goalkeepers from the aforementioned tournament was used. Random probability sampling was implemented: in this type of sampling, all the individuals of the population may take part in the sample, with a positive probability of participation. Therefore, it was the proper sampling type for the development of the present investigation.

\section{Variables}

Variable: measurement instrument.

Variable: interception velocity.

Variable: measurement instrument

The technological platform that permits the collection of the measurements of the ball interception velocity of the goalkeepers is composed of three elements: a shot detection platform, a cinematic data capture system 
and the software. The first element is located on the ground, at the penalty point and in front of the goal, the second element has to be placed on the forearm and upper arm of the goalkeeper, and the last element to one side of where the test is executed, and there the control of the information is carried out, sending a digital signal to the computer, where it is processed.

Variable: interception velocity

The ball interception velocity variable is given by the effectiveness of the interception, by the right or left segments. Measuring this variable permits an analysis, averaging and characterisation of each of the participants according to their performance range.

\section{Materials used}

The materials used were the following: leather footballs with a circumference of 60-62 cm, with $440 \mathrm{~g}$ to 450 $\mathrm{g}$ of weight, and a calibration of 9 pounds of pressure. The same balls were used in each test. Number of balls: 5 . In addition, the following instruments were used:

- Camera (handycam).

- Platform to measure interception speed.

- Data sheet.

\section{Test scenario}

The tests were carried out on a football pitch that complied with the rules of futsal. The instruments for the capturing of data were put in place in advance, in front of and longitudinally to the goal, at a distance of 6 meters. The surfaces where the tests were conducted were: wood (coliseum) and tile (outdoor pitch). When the tests took place, 5 people participated, who had different tasks within the application of the study.

- Goalkeeper: individuals from whom the information was collected.

- Kicker: the same player executed all the shots.

- Recording and photographic record: individual in charge of taking photographs and filming each one of the tests.

- Note taker: the person who kept a manual record of the information of each one of the goalkeepers

- Software: Management of the captured data issued by the platform.

\section{Procedure}

In the process of this investigation, four phases can be identified. They are aimed at obtaining information that permits the evaluation of certain factors that contribute to the control process and follow-up of the training of the futsal goalkeeper. Said phases are:

- Phase 1: Elaboration of a platform that allows the measurement of the ball interception velocity by futsal goalkeepers.

- Phase 2: Application of the tests to sportsmen from the Copa Hero Claro de Fútbol de Salón in Colombia.

Before starting the tests, the following aspects have to be taken into consideration and included:

- Specific clothing for the goalkeeper.

- General and specific warm up for goalkeepers (15-20 minutes).

- Placement of the motion capture sensors on the goalkeepers' upper limbs.

- Testing shots to calibrate the instruments.

- Goalkeeper's familiarization with the test.

- Location of the goalkeeper (feet on the goal line and in the basic position).

$\circ \quad$ Shot distance (6 meters). 
- Start the test by taking frontal shots with a stationary ball, at random, and registered on data sheet.

End of the test and stretching.

- Phase 3: Analysis of the data obtained when applying the tests and captured by IMOCAP.

- Phase 4: Creation of document to analyse the performance of the goalkeepers participating in the tournament.

\section{RESULTS}

In order to carry out the analysis of the study variable (interception velocity), it was necessary to develop a technological platform that automatised the data collection process. The platform to measure the ball interception velocity is a complete system that provides relevant information about the movement of the ball and the basic characterization at the moment of interception. This set of characteristics includes various electronic and software elements, which make the detection of the ball possible, from the moment that it is in the initial shot position up to its interception by the goalkeeper.

\section{Interception velocity motion capture platform}

The platform is composed of three elements which permit the detection of the ball, the collection of the data of the shot, and its interception. The first element, called "shot detection platform", which perceived the initial position of the ball (before the shot) and which activates when the ball leaves the detection platform, located on the ground of the futsal pitch. The second element, "Capture system IMOCAP" is used to detect the interception of the ball in the goal area. This system is a set of sensors, processors, and communication modules which are placed on both of the goalkeeper's arms. Finally, the third element is a computer application (software) which integrates the information of the two previous elements, carries out mathematical calculations, saves different parameters into record files, and presents the information in an orderly, clear and precise manner, where the characteristics of the execution of the shot are shown. More information in Callejas-Cuervo et al. (2018, p.28).

\section{Anthropometric characterisation of the group in the study}

Before the realisation of the tests on the goalkeepers, the sample, constituted by 10 goalkeepers, is characterised. This exploratory analysis includes: age, weight, height, body mass index (BMI), right arm length, and left arm length. The results are presented in Table 1. Measurements were obtained by processing the data of the variables using SPSS software.

Table 1. Age of the goalkeepers participating in the study.

\begin{tabular}{lccccc}
\hline Item & N & Minimum & Maximum & Average & Standard Deviation \\
\hline Age (years) & 10 & 19 & 31 & 27.7 & 3.74 \\
Height (in meters) & 10 & 1.66 & 1.87 & 1.74 & 0.07 \\
Weight (kilograms) & 10 & 68 & 108 & 77.90 & 11.97 \\
Body mass index & 10 & 21.60 & 30.88 & 256.340 & 2.49 \\
Right arm length (centimetres) & 10 & 71.00 & 81.00 & 75.10 & 3.37 \\
Left arm length (centimetres) & 10 & 70.00 & 80.00 & 74.75 & 3.19 \\
\hline
\end{tabular}

In summary, from the descriptive analysis it is deduced that the group in the study, formed by 10 participating professional goalkeepers is characterised as being homogenous in the data corresponding to the variables of height, right arm length, and left arm length. In the same way, the goalkeepers are similar or nearly homogenous as regards age, weight and BMI. In consequence, the goalkeepers in the study are quite similar 
with respect to anthropometric characterisation. These results allow a better analysis of the correlation of these variables with the ball interception velocity of the goalkeepers.

\section{Characterisation of the shots}

The shots taken in order to carry out the test of the futsal goalkeepers were supervised by a group of researchers. They were recorded and the information was registered manually in data sheets. In the data sheet, it was registered if there was an interception, or not, of the ball by the goalkeeper, and in which quadrant. This was carried out in addition to what was registered by the software in order to corroborate the information. Forty consecutive shots were taken with pauses between each shot. The goalkeeper received approximately the same number of shots until reaching ten effective shots (with a validated interception) for each of the upper limbs. The above process was carried out taking into account the process stipulated in the methodology, referring to the realisation of the tests.

\section{Interpretation of the results of the data obtained using the measurement platform}

In this section a descriptive analysis is made of the variables: velocity towards the upper right limb and velocity towards the upper left limb, which contain data expressed in units of kilometres per hour $(\mathrm{km} / \mathrm{h})$.

In Table 2, anthropometric data can be observed which characterises the group of goalkeepers in this study and the variables related to the ball interception velocity by the goalkeepers on each of their sides.

Table 2. Velocity to the right and velocity to the left of the goalkeepers.

\begin{tabular}{lcccccccc}
\hline Subject No. & Age & Height & Weight & BMI & $\begin{array}{c}\text { Right arm } \\
\text { length }\end{array}$ & $\begin{array}{c}\text { Left arm } \\
\text { length }\end{array}$ & $\begin{array}{c}\text { Velocity right } \\
\text { side }\end{array}$ & $\begin{array}{c}\text { Velocity left } \\
\text { side }\end{array}$ \\
\hline S1 & 23 & 1.66 & 76 & 27.58 & 72 & 73 & 120.45 & 80.32 \\
S2 & 19 & 1.8 & 70 & 21.6 & 76 & 75 & 100.87 & 108.1 \\
S3 & 31 & 1.76 & 83 & 26.79 & 76 & 77.5 & 71.91 & 115.53 \\
S4 & 27 & 1.87 & 108 & 30.88 & 81 & 80 & 86.26 & 86.23 \\
S5 & 23 & 1.82 & 85 & 25.66 & 78 & 77 & 85.39 & 92.9 \\
S6 & 26 & 1.69 & 71 & 24.86 & 71 & 70 & 58.72 & 91.17 \\
S7 & 20 & 1.7 & 68 & 23.53 & 72 & 73 & 60.65 & 43.55 \\
S8 & 27 & 1.71 & 73 & 24.96 & 74.5 & 73.5 & 77.28 & 47.16 \\
S9 & 28 & 1.7 & 75 & 25.95 & 72 & 71 & 49.88 & 49.93 \\
S10 & 23 & 1.69 & 70 & 24.51 & 78.5 & 77.5 & 49.75 & 51.47 \\
\hline
\end{tabular}

From the information registered in Table 2, it is deduced that two goalkeepers, subjects S1 and S2, register a velocity towards the upper right of $100 \mathrm{~km} / \mathrm{h}$, four goalkeepers present a velocity to the right of between 70 and $100 \mathrm{~km} / \mathrm{h}$ (S3, S4, S5 and S8) and four goalkeepers reach a ball interception velocity below 70km/h (S6, S7, S9 and S10). In addition, two goalkeepers register a velocity to the left of over $100 \mathrm{~km} / \mathrm{h}$ (S2 and S3), four goalkeepers demonstrate a velocity to the left of between 70 and $100 \mathrm{~km} / \mathrm{h}$ (S1, S4, S5, and S6) and four goalkeepers reach a ball interception velocity of less than $70 \mathrm{~km} / \mathrm{h}(\mathrm{S} 7, \mathrm{~S} 8, \mathrm{~S} 9$, and S10). Upon analysing the results individually, it is possible to create a first approximation of the performance of the goalkeepers with respect to the interception velocity. In this way, the results can allow decisions to be taken with respect to the design of sports training strategies which can improve the individual and global performance of goalkeepers in this sport.

Below is presented a group descriptive analysis associated with the variables: the velocity to the right and the velocity to the left of the goalkeepers when intercepting the ball. 
In relation to the global results, reference is made to the velocity to the right of all of the goalkeepers. It is established that this velocity is between 49.75 and $120.45 \mathrm{~km} / \mathrm{h}$. The group showed an average velocity to the right of 76.116 with a standard deviation of $=22.88$. The coefficient of variation (CV) is $22.88 / 76.116=$ $0.3005=30.05 \%$. This percentage indicates that the goalkeepers in this group are heterogenous as regards the velocity to the right; that is, these goalkeepers have little in common with respect to velocity to the right. The variability in this characteristic is produced because two of the goalkeepers have a velocity above $100 \mathrm{~km} / \mathrm{h}$ and another two have a velocity close to $49 \mathrm{~km} / \mathrm{h}$, which are far from the mentioned average velocity.

In a similar way, regarding velocity to the left, it is established that this velocity is found to be between 43.55 and $115.53 \mathrm{~km} / \mathrm{h}$. The group under study had an average velocity to the right of 76.636 , with a standard deviation of $\pm 26.6544 \mathrm{~km} / \mathrm{h}$; the coefficient of variation (CV) is $34.78 \%$, indicating that the goalkeepers exhibit great differences with respect to the velocity to the left. The variability in these characteristics is attributed to the fact that two goalkeepers had a velocity of over $100 \mathrm{~km} / \mathrm{h}$ and one presented a velocity close to $43 \mathrm{~km} / \mathrm{h}$. These velocities are far from the average of the velocity to the left.

From the values in Table 3, it is deduced that the data of the velocity to the left are normal, given that the $p$ value (Sig) of .977 is greater than .05. At the same time, the data of the velocity to the left are also normal given that the p-value (Sig) of.679 is greater than 0.05 . Therefore, it fulfils the assumptions of normality. As in this case there are independent samples, it is feasible to apply a Student's t-test, which has been applied with a significance level of $\alpha=.05$.

Table 3. Kolmogorov-Smirnov Test for velocities.

\begin{tabular}{|l|l|c|c|}
\hline \multicolumn{2}{|l|}{ Item } & Velocity to the right & Velocity to the left \\
\hline $\mathrm{N}$ & 10 & 10 \\
\hline \multirow{2}{*}{ Normal Parameters(a,b) } & Mean & 761.160 & 766.360 \\
\cline { 2 - 4 } & Std. Deviation & 2.288 .019 & 2.665 .448 \\
\hline \multirow{3}{*}{ Most Extreme Differences } & Absolute & .150 & .227 \\
\cline { 2 - 4 } & Positive & .150 & .227 \\
\cline { 2 - 4 } & Negative & -.125 & -.155 \\
\hline Kolmogorov-Smirnov Z & .476 & .719 \\
\hline Asymp. Sig. (2-tailed) & .977 & .679 \\
\hline
\end{tabular}

Note: a Test distribution is Normal. b Calculated from data.

\section{Creation of categories}

With the purpose of classifying the goalkeepers, the following scales have been proposed, constructed based on the ranges of the velocity to the left or to the right, respectively. The first scale includes the following categories: a) Low, when the velocity to the right is between 30 and $75 \mathrm{~km} / \mathrm{h}, \mathrm{b}$ ) Medium, when the velocity to the right ranges from 75 to $119 \mathrm{~km} / \mathrm{h}$, and Fast, when the velocity is above $119 \mathrm{~km} / \mathrm{h}$. The second scale includes the following categories: a) Low, when the velocity to the left is between 32 and $75 \mathrm{~km} / \mathrm{h}$, b) Medium, when the velocity to the left ranges from 75 to $119 \mathrm{~km} / \mathrm{h}$, and Fast, when the velocity is above $119 \mathrm{~km} / \mathrm{h}$.

In accordance with the two above scales, referring to the velocities, the following results were obtained:

1. Out of the goalkeepers who participated in the study, $50 \%$ were placed in the Low category; that is, their interception velocity to the right is low; $40 \%$ of the goalkeepers were placed in the medium category; that is, their interception velocity to the right is moderate; only $10 \%$ of the goalkeepers were placed in the Fast category; that is, their interception velocity was high (see Table 4). 
Table 4. Velocity to the right scale.

\begin{tabular}{llccc}
\hline \multicolumn{2}{c}{ Item } & Frequency & Valid Percent & Cumulative Percent \\
\hline \multirow{3}{*}{ Validation } & Low & 5 & 50.0 & 50.0 \\
& Medium & 4 & 40.0 & 90.0 \\
& Fast & 1 & 10.0 & 100.0 \\
\hline Total & & 10 & 100.0 & \\
\hline
\end{tabular}

2. According to the data in Table 5 referring to the velocity to the left, $40 \%$ of the goalkeepers participating in the study were placed in the Low category; that is, their interception velocity is low, whereas $60 \%$ of them were placed in the Medium category; that is, their interception velocity is moderate. None of the goalkeepers were placed in the Fast category.

Table 5. Velocity to the left scale.

\begin{tabular}{lcccc}
\hline \multicolumn{2}{c}{ Item } & Frequency & Valid Percent & Cumulative Percent \\
\hline \multirow{2}{*}{ Validation } & Low & 4 & 40.0 & 40.0 \\
& Medium & 6 & 60.0 & 100.0 \\
\hline Total & & 10 & 100.0 & \\
\hline
\end{tabular}

In summary, when comparing the velocities, it could be established that the average velocity to the right is slightly lower than the average velocity to the right. The hypothesis testing led to the conclusion that there are differences between the data received regarding velocity to the left and velocity to the right. However, the differences are not significant in the goalkeepers of the study group, using a level of $p<.05$.

\section{Correlational analysis of velocities to the left and to the right}

With the purpose of analysing the relationships and the strength of the correlations between the length of the arms and the ball interception velocity variables, a Pearson correlation matrix was used that is presented in Table 6. Additionally, the correlations between height, the body mass index (BMI) and the ball interception velocity were calculated in order to detect the existence of other correlations.

Every value different from 1 in the correlation matrix corresponds to a Pearson correlation coefficient. This value was calculated with the data of the variables of interest that are indicated in that matrix. A value close to 1 in the correlation coefficient $r$ is indicative of the existence of a strong and direct correlation between the variables involved. A value close to 0.5 indicates the existence of a moderate and direct relationship; a value close to 0 but with a positive value signifies a weak and direct relationship; a value close to 0 with a negative value indicates a weak and inverse relationship. A value close to -0.5 indicates a moderate and inverse relationship, and a value close to -1 indicates a strong and inverse relationship. In Table 6, the correlations between the variables of interest of the goalkeepers participating in this study can be seen.

To interpret the results of the correlation matrix, it is noted that: if the p-value Sig. (2-tailed) is greater than $.05=5 \%$ called the level of significance, therefore, there exists a correlation between the variables, but not a significant one. On the contrary, if the p-value Sig. (2-tailed) is less than $.05=5 \%$, then the correlation is significant.

In accordance with the results seen in Table 6, it is deduced that between the length of the right arm and the velocity to the right, there is a correlation of $r=.112$ with a $p$-value Sig. (2-tailed) of .757 , which is greater than .05 . These results indicate that between the length of the right arm and the velocity to the right there is 
a weak and direct relationship but which is not significant. That is, a slight increase in the length of the right arm implies a slight increase in the velocity to the right, and vice versa.

Table 6. Correlation matrix in the group of goalkeepers.

\begin{tabular}{|c|c|c|c|c|c|c|c|}
\hline 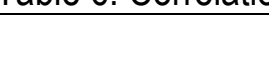 & tem & Right arm & Left arm & Speed right & Speed left & Height & BMI \\
\hline & Pearson Correlation & 1 & $.950^{\star \star}$ & .112 & .264 & .774 & .367 \\
\hline Right arm & Sig. (2-tailed) & & .000 & .757 & .461 & .009 & 297 \\
\hline & $\mathrm{N}$ & 10 & 10 & 10 & 10 & 10 & 10 \\
\hline & Pearson Correlation & $.950^{* *}$ & 1 & .190 & .328 & $.720^{*}$ & .423 \\
\hline Left arm & Sig. (2-tailed) & 0 & & .599 & .354 & .019 & .223 \\
\hline & $\mathrm{N}$ & 10 & 10 & 10 & 10 & 10 & 10 \\
\hline & Pearson Correlation & .112 & .190 & 1 & .477 & .263 & .198 \\
\hline Right arm & Sig (2-tailed) & .757 & .599 & & .164 & .463 & .582 \\
\hline & $\mathrm{N}$ & 10 & 10 & 10 & 10 & 10 & 10 \\
\hline & Pearson Correlation & .264 & .328 & .477 & 1 & .528 & .124 \\
\hline Left arm & Sig (2-tailed) & .461 & .354 & .164 & & .117 & .732 \\
\hline & $\mathrm{N}$ & 10 & 10 & 10 & 10 & 10 & 10 \\
\hline & Pearson Correlation & $.774^{* \star}$ & .720 & .263 & .528 & 1 & .322 \\
\hline Height & Sig (2-tailed) & .009 & .019 & .463 & .117 & & .365 \\
\hline & $\mathrm{N}$ & 10 & 10 & 10 & 10 & 10 & 10 \\
\hline & Pearson Correlation & .367 & .423 & .198 & .124 & .322 & 1 \\
\hline Body mass index & Sig (2-tailed) & 297 & 223 & .582 & .732 & .365 & \\
\hline & $\mathrm{N}$ & 10 & 10 & 10 & 10 & 10 & 10 \\
\hline
\end{tabular}

Note: ${ }^{*}$ Correlation is significant at the 0.05 level (2-tailed). ${ }^{* *}$ Correlation is significant at the 0.01 level (2-tailed).

From Table 6, it can also be deduced that between the length of the right arm and the velocity to the right there is a correlation of $r=.264$ with a p-value Sig. (2-tailed) of .461, which is greater than .05 . These results show that between the length of the right arm and the velocity to the right there is a moderate and direct relationship but not a significant one. That is, a moderate increase in the length of the right arm implies a moderate increase in the velocity to the left, and vice versa.

On the other hand, according to the results shown in Table 6, it can be deduced that between the length of the left arm and the velocity to the right, there is a correlation of $r=.190$ with a $p$-value Sig. (2-tailed) of .599, which is greater than .05. These results show that between the length of the left arm and the velocity to the right there is a weak and direct relationship, but not a significant one. That is, a slight increase in the length of the left arm implies a slight increase in the velocity to the right, and vice versa.

Based on Table 6, it is deduced that between the length of the left arm and the velocity to the left there is a correlation of $r=.328$ with a p-value Sig. (2-tailed) of .354, which is greater than .05 . These results indicate that between the length of the left arm and the velocity to the left there is a moderate and direct relationship, but it is not significant. In other words, a slight increase in the length of the left arm generates a moderate increase in the velocity to the left, and vice versa.

From Table 6, it can be deduced that between height and the velocity to the right, there is a correlation of $r=$ .263 with a p-value Sig. (2-tailed) of .463, which is greater than .05 . These results indicate that between 
height and the velocity to the right there is a moderate and direct relationship, but not a significant one. That is, a moderate increase in height implies a moderate increase in the velocity to the right, and vice versa.

Based on Table 6, it is inferred that between height and velocity to the left there is a correlation of $r=.528$ with a p-value Sig. (2-tailed) of .117, which is greater than .05. In consequence, these results show that between height and velocity to the left there is a moderate and direct but not significant relationship. That is, a moderate increase in height implies a moderate increase in the velocity to the left, and vice versa.

From the information presented in Table 6 , it can be deduced that between BMI and velocity to the right there is a correlation of $r=.198$ with a p-value Sig. (2-tailed) of .582. These results indicate that between BMl and velocity to the right there is a weak and direct relationship, although not a significant one. That is, a slight decrease in BMI implies an increase in the velocity to the right, and vice versa.

Based on Table 6, it can be inferred that between BMI and velocity to the left there exists a correlation of $r=$ .124 with a p-value Sig. (2-tailed) of .732, which is greater than .05. In consequence, these results indicate that between BMI and velocity to the left there is a weak and direct, but not significant relationship. That is, a slight increase in BMI implies a slight decrease in the velocity to the left, and vice versa.

Below, the significant correlations are analysed. Between height and the length of the right arm there is a significant correlation, as the Pearson correlation coefficient is $r=.774$ and the $p$-value Sig. (2-tailed) of .009 is less than .05. Therefore, there is a strong and direct correlation which indicates that an increase in height implies a marked increase in the length of the right arm of the goalkeepers, and vice versa. Also, between height and the length of the left arm there is a significant correlation as the Pearson correlation coefficient is $r=.720$ and the p-value Sig. (2-tailed) is less than .05. Therefore, there is a moderate and direct correlation, which indicates that an increase in height generates a moderate increase in the length of the left arm of the goalkeepers, and vice versa.

Now the other non-significant correlations are analysed. Between BMI and the length of the right arm there is a non-significant correlation given that the Pearson correlation coefficient is $r=.367$ and the $p$-value Sig. (2-tailed) of .297 is greater than .05. In consequence, there is a moderate and direct correlation. This indicates that an increase in the BMI implies a moderate increase in the length of the right arm of the goalkeepers, and vice versa. Also, between the BMl and the length of the left arm there exists a non-significant correlation in that the measure of the Pearson correlation coefficient is $r=.423$ and the p-value Sig. (2-tailed) of .223 is greater than .05. Thus, there is a moderate and direct correlation, which indicates that an increase in the BMI implies a moderate increase in the length of the left arm of the goalkeepers, and vice versa.

In synthesis, the length of the arms weakly and directly affects the velocity to the right and moderately and directly the velocity to the left, although not significantly. In addition, height influences velocity to the right and the left, moderately and directly, although not in a significant way. In contrast, BMI weakly and directly affects the velocities, but not significantly.

Finally, between height and the length of the right arm there is a strong, direct and significant relationship, in that between height and the length of the left arm, the relation is significant, moderate and direct. In contrast, between the BMl and the length of the arms there is a direct, moderate but not significant correlation. 


\section{DISCUSSION}

One of the aspects most commonly studied are the anthropometric measurements. For example, this study showed that the BMI of the goalkeeper directly influences their particular functions and are similar in respect to the ball interception velocity. According to the results from the right and left limbs of the goalkeepers and, in some other studies, such as those proposed by Nuñez et al. (2004), the right limb demonstrates a better performance, establishing differences, although not significant ones, according to the length of the arms. This weakly and directly affects the velocity to the right, and moderately and directly the velocity to the left, establishing the dominant hand of each of the goalkeepers in the study sample.

The study proposed by Antúnez (2003), shows, in their observation, relevant aspects of the behaviour of female handball goalkeepers in actions of real games, with the application of a perceptual-motor training plan and its positive effect on the development of the same. Beginning from the actions of the experimental and control goalkeeper, represented in her efficiency and evolution of the motor behaviour in the carrying out of tasks particular to this position, making emphasis on the goalkeeper that implemented the training plan. Therefore, the work designed in the analysis of the ball interception, using IMOCAP describes the influence of arm length and the direct and weak effect of the right arm and the moderate effect of the left arm, in such a way that it is pertinent to implement intervention factors which directly influence the physical part, as well as the visual, in order to complement the training of these subjects.

Martínez (2003), in their analysis of visual reaction time in karate, found, as a result, that reaction time is not an automatic condition that takes place with movement and effort, but is rather determined by the characteristics of the task. In this way, it is presented that the ball interception movement is directly influenced according to the right or left arm segment used in the execution of specific ball interception actions and the dominant hand of the subject.

In Suarez (2010), with the design of new measurement instruments to carry out the continuous work of these tests, it allows specific scales to be made to evaluate related variables. The interrelated correlation coefficients were between .99 and .88 , establishing that the indicators were trustworthy in the measurement of execution time and movement time, allowing the diagnostics to be generated of how the group scored in terms of gesture speed, a determining guide to performance in order to be able to make the respective corrections to the training, establishing movement execution ranges. In this way, the proposed study establishes a categorisation with respect to the ball interception velocity to the right, Low (between 30 and $75 \mathrm{~km} / \mathrm{h}$ ); Medium (between 75 and $119 \mathrm{~km} / \mathrm{h}$ ) and Fast (more than $119 \mathrm{~km} / \mathrm{h}$ ), in addition to, with respect to the velocity to the left, Low (between 32 and $75 \mathrm{~km} / \mathrm{h}$ and Medium (75 to $199 \mathrm{~km} / \mathrm{h}$ ). These results classify the range of the best performances of the goalkeepers in the velocity to the right with an instrument that ratifies its validity according to the results found by the IMOCAP device platform.

This research is concerned with the specific task of a futsal goalkeeper, the role of the goalkeeper being vital for a good performance on the pitch. They make an exercise plan to improve execution speed in anticipation of the ball, in which the goalkeeper shows improvement, in our case we analysed the interception velocity of the goalkeepers which provided data in order to determine their mistakes.

Regarding the study of the assessment of the motor behaviour and movement pre-indicators of the futsal goalkeepers during the taking of a penalty, proposed by Núñez et al. (2004) and what is reported here, there are similarities with respect to the reaction of the goalkeepers The difference is that in the study by Núñez et al. (2004), they used a digital video camera and predicted the movement of the goalkeepers according to the 
angle of the inclination of the knee and in this study the IMOCAP motion capture system was used, which analyses the interception velocity of the ball by the goalkeepers.

Nevertheless, studies have not been found which have determined a more detailed analysis of the ball interception velocity in futsal goalkeepers. Thus, the model that has been generated in this investigation responds to the basic necessity of incorporating technological instruments which measure in an objective and efficient manner the assessment of special techniques for futsal goalkeepers, in this case regarding ball interception velocity.

\section{CONCLUSIONS}

From the descriptive analysis, it is deduced that the goalkeeper sample group are homogenous with respect to the characteristics of height, and right and left arm length. The goalkeepers were somewhat similar as regards age, weight and BMI. Therefore, the goalkeepers are similar in terms of anthropometric characteristics related to ball interception velocities.

A descriptive analysis allowed the classification of the group of goalkeepers in the study in the following categories: with respect to the ball interception velocity to the right, $50 \%$ of the goalkeepers fell into the Low range; $40 \%$ in the Medium range, and the remaining $10 \%$ in the Fast range. Also, with respect to the velocity to the left, $40 \%$ were located in the Low range and the remaining $60 \%$ in the Medium range. These results classify the range of the best performance of the goalkeepers in the velocity to the right.

By means of a comparative study, it is established that the average velocity to the right is slightly lower than the velocity to the left. Nevertheless, the goalkeepers are less heterogenous in the velocity to the right. A significance test of $5 \%$ concluded that there are differences between the data regarding the velocity to the left and the velocity to the right, but the differences were not significant on average with respect to the group of goalkeepers in the study.

Based on the Pearson correlation matrix, it was determined that: the arm length had a weak, direct effect on the velocity to the right and a moderate, direct effect on the velocity to the left. Also, height influenced the velocities to the right and the left in a direct and moderate way, although these correlations were not significant. Finally, the BMI affects the velocities weakly and directly, but not significantly

When analysing other correlations, the following were found: a strong, direct and significant correlation between height and right arm length, a moderate, direct and significant relationship between height and left arm length, and a moderate, direct but not significant correlation between BMI and the arm length of the goalkeepers.

The validation of the IMOCAP instrument is provided by publications in scientific journals and is an instrument which provides reliability for the acquisition of data, in this case of interception velocity, Callejas-Cuervo et al. (2017), Callejas Cuervo et al (2016).

\section{REFERENCES}

Antúnez, A. (2003). La inteceptacion en la portera de balonmano: Efectos de un programa de entrenamiento perceptivo-motriz. (Tesis Doctoral), Universidad de Murcia, Murcia, España. 
Callejas-Cuervo, M.; Gutierrez, R. M.; Hernandez, A.I. (2017). Joint amplitude MEMS based measurement platform for low cost and high accessibility telerehabilitation: Elbow case study. Journal of Bodywork and Movement Therapies. Volume 21, Issue 3, 2017, p 574-581. https://doi.org/10.1016/j.jbmt.2016.08.016

Callejas-Cuervo, M., Ruíz-Olaya, A.F. and Gutiérrez, R.M., Validation of an inertial sensor-based platform to acquire kinematic information for human joint angle estimation. DYNA 83(197), pp. 154$159,2016$.

Carlos-Vivas, J.; Freitas, T.; Cuesta, M.; Pérez Gómez, J.; de Hoyo Lora, M.; Alcaraz Ramón, P.E. (2019). New Tool to Control and Monitor Weighted Vest Training Load for Sprinting and Jumping in Soccer. Journal of strength and conditioning research: the research journal of the NSCA, ISSN 10648011, Vol. 33, No. 11, 2019, págs. 3030-3038. https://doi.org/10.1519/jsc.0000000000002580

Estrada Ruiz, L.I. (2019). Determinación y comparación de indicadores de demandas físicas en partidos y entrenamientos en fútbol profesional femenil mediante uso de tecnología GPS. Tesis de master Universidad Autónoma de Nuevo León. Julio 2019.

Ferley, Derek D.; Scholten, Shane; Vukovich, Matthew D. Combined Sprint Interval, Plyometric, and Strength Training in Adolescent Soccer Players: Effects on Measures of Speed, Strength, Power, Change of Direction, and Anaerobic Capacity, Journal of Strength and Conditioning Research: April 2020 - Volume 34 - Issue 4 - p 957-968. https://doi.org/10.1519/JSC.0000000000003476

Martínez, O. (2003). Tiempo de reaccion visual en el karate. (Tesis Doctoral), Universidad Politécnica de Madrid, Madrid,España.

McFadden, Bridget A.1; Walker, Alan J.2; Bozzini, Brittany N.1; Sanders, David J.3; Arent, Shawn M.1,3 Comparison of Internal and External Training Loads in Male and Female Collegiate Soccer Players During Practices vs. Games, Journal of Strength and Conditioning Research: April 2020 - Volume 34 - Issue 4 - p 969-974. https://doi.org/10.1519/jsc.0000000000003485

Navia, J. (2013). Estrategias visuales y motrices de los porteros expertos de futbol sal en tareas de interceptacion bajo precion temporal y espacial. (Tesis Doctoral), Universidad Politécnica de Madrid, Madrid, España.

Nuñez, F. J., Bilbao, A., Raya, A., \& Oña, A. (2004). Valoracion del comportamiento motor y preindices delmovimiento del portero de futbol durante el lanzamiento de penalti. European Journal of Human Movement, 12, 21-38.

Núñez, F. Javier; Toscano-Bendala, Francisco J.; Suarez-Arrones, Luis; Martínez-Cabrera, F. Ignacio; De Hoyo, Moisés. (2019). Individualized thresholds to analyze acceleration demands in soccer players using GPS. Retos: nuevas tendencias en educación física, deporte y recreación, ISSN 15791726, No. 35, p 75-79.

Suarez, E. (2010). Diseño y aplicación de nuevos instrumentos específicos de evaluación para medir las distintas manifestaciones de la velocidad en la técnica Bandal Chagui (patada semicircular) de los deportistas juveniles de la provincia de Cotopaxi. (Trabajo de maestria), Escuela politecnica del Ejercito, sangolquí, Ecuador.

\section{() $(\Theta \Theta \Theta$}

This work is licensed under a Attribution-NonCommercial-NoDerivatives 4.0 International (CC BY-NC-ND 4.0). 